\title{
Meta-analysis of the prognostic value of p-4EBP1 in human malignancies
}

\author{
Tao Zhang ${ }^{1}$, Jianrong Guo ${ }^{1}$, Huili Li $^{1}$ and Jiliang Wang ${ }^{1}$ \\ ${ }^{1}$ Department of Gastrointestinal Surgery, Union Hospital, Tongji Medical College, Huazhong University of Science and \\ Technology, Wuhan, 430022, Hubei, China \\ Correspondence to: Huili Li, email: huili_li@hust.edu.cn \\ Jiliang Wang, email: jiliang_wang@hust.edu.cn
}

Keywords: $p-4 E B P 1$; prognosis; cancer; meta-analysis; malignancies

Received: July 02, $2017 \quad$ Accepted: September 20, $2017 \quad$ Published: December 07, 2017

Copyright: Zhang et al. This is an open-access article distributed under the terms of the Creative Commons Attribution License 3.0 (CC BY 3.0), which permits unrestricted use, distribution, and reproduction in any medium, provided the original author and source are credited.

\begin{abstract}
Phosphorylated 4E-binding protein 1 (p-4EBP1) is the inactivated form of 4EBP1, which is a downstream mediator in the $\mathrm{mTOR}$ signaling pathway and a vital factor in the synthesis of some oncogenic proteins. This meta-analysis was conducted to assess the predicative value of p-4EBP1 expression in human malignancies. The PubMed and Embase databases were carefully searched. Articles comparing the prognostic worthiness of different p-4EBP1 levels in human malignancies were collected for pooled analyses and methodologically appraised using the Newcastle-Ottawa Scale (NOS). A total of 39 retrospective cohorts with an overall sample size of 3,980 were selected. Patients with lower p-4EBP1 expression had better 3 -year $(P<0.00001)$, 5-year $(P<0.00001)$, and 10-year $(P=0.03)$ overall survival and better 3 -year $(P<0.0001)$ and 5-year $(P=0.0005)$ disease-free survival. Subgroup analyses confirmed the unfavorable prognosis associated with p-4EBP1 overexpression. These findings were further validated by sensitivity analyses. Harbord and Peters tests revealed no publication bias within the included studies. It thus appears higher expression of p-4EBP1 indicates a poor prognosis in human malignancies.
\end{abstract}

\section{INTRODUCTION}

Eukaryotic translation initiation factor $4 \mathrm{E}$ binding protein 1 (4EBP1) is a family of translation-repressor proteins, which is one of the two main downstream effectors (4EBP1and S6K1) of mammalian target of rapamycin (mTOR) [1]. 4EBP1 inhibits protein synthesis by binding to eIF4E. Dephosphorylated active 4EBP1 binds to eukaryotic translation initiation factor $4 \mathrm{E}$ (eIF4E) and disrupts the formation of the cap-dependent translation initiation complex, comprising the eIF4A RNA helicase, the eIF4E mRNA cap-binding protein, and the eIF4G scaffolding protein, which is essential for protein synthesis and is associated with cancer development and progression [2]. When 4EBP1 is phosphorylated and deactivated by upstream signals at several sites (Thr37/46, Thr70, Ser65, etc.), eIF4E is released from 4EBP1 and initiates cap-dependent translation to promote the synthesis of various proteins, including oncogenic proteins [3].
4EBP1 is a tumor suppressor and its deactivation (phosphorylation) might directly contribute to the development of multiple cancers [4]. Research have shown that loss of 4EBP1 function (phosphorylation of 4EBP1) can induce epithelial-mesenchymal transformation, migration, and invasion by cancer cells [5]. Accordingly, the expression of p-4EBP1 in tumor cells might indicate their oncogenic potential. Furthermore, studies have shown that the expression of $\mathrm{p}-4 \mathrm{EBP} 1$ in tumor tissues is a predictive biomarker of malignant potential.

However, despite the accumulating of evidence in the literature, the prognostic value of $\mathrm{p}-4 \mathrm{EBP} 1$ remains controversial. Benavente et al. [6] showed that a high level of p-4EBP1 correlates with a worse prognosis in patients with cervical cancer. Lee et al. [7] believed that gastric cancer patients with overexpression of p-4EBP1 had a prolonged survival time compared with low-expression patients. Azim et al. [8] reported that expression of p-4EBP1was not associated with prognosis in breast cancer patients. 
To evaluate the prognosis and predictive value of the expression of p-4EBP1 in human malignancies, we conducted a comprehensive meta-analysis to provide theoretical support for clinical applications.

\section{MATERIALS AND METHODS}

This meta-analysis was performed according to Cochrane Collaboration protocols and the PRISMA Checklist. Two investigators independently performed each step, and any disagreement was resolved by mutual discussion.

\section{Literature search}

The Pubmed and Embase databases were searched by use of the terms (EIF4EBP1 protein OR Phosphorylated 4E-binding protein 1 OR p-4E-BP1 OR 4E-BP1 OR 4E-binding protein 1) AND (cancer OR carcinoma OR malignancy OR tumor). Abstracts and full-text of the preliminary articles were screened in sequence to ensure the eligibility of included articles.

\section{Selection criteria}

The inclusion criteria were as follows: (1) Englishlanguage articles formally published until May 2017 and (2) studies that compare the prognostic value of different p-4EBP1 expressions in human malignancies. The elimination criteria were as follows: (1) Duplicated or overlapped articles, (2) review articles and case reports, and (3) articles with insufficient original data on survival analysis. Two investigators performed each evaluation independently to ensure the accuracy of the selection process.

\section{Data extraction}

A standardized form was designed for the data-extraction process. Original data of elementary demographic characteristics (country, tumor type, TNM stage, detection method, p-4EBP1 level, phosphorylation site, subcellular localization, sample size, sex, age, and follow-up duration) and survival data (3-year overall survival, 5-year overall survival, 10-year overall survival, 3-year disease-free survival, 5-year disease-free survival, and 10-year disease-free survival) were extracted from text words, tables, or Kaplan-Meier curves. Survival data were mainly obtained from Kaplan-Meier curves and digitized by GetData Graph Digitizer 2.25 software.

\section{Methodological assessment}

The Newcastle-Ottawa Scale (NOS) was utilized for methodological appraisal of the included observational studies. Three categories, including selection, comparability, and outcome, were established within the scale, with a maximum score of nine. Studies with scores of six or higher were graded as high quality .

\section{Statistical analysis}

Statistical procedures were performed by Review Manager 5.3 software. The effect size was presented by odds ratio, and the Mantel-Haenszel model was utilized for dichotomous variables. The $\mathrm{I}^{2}$ value was adopted as a heterogeneity indicator among the included studies, and $<25 \%,<50 \%$, and $>50 \%$ implied low, moderate, and severe heterogeneity, respectively. A fixed-effects model was adopted for low heterogeneity, and a random-effects model was utilized for the remaining conditions. $P<0.05$ indicated a statistical significance within the comparisons. Subgroup analyses were additionally conducted to explore potential confounding elements. The consistency of pooled outcomes was evaluated by sensitivity analysis. Publication bias was analyzed by the Harbord test and the Peters test.

\section{RESULTS}

\section{General characteristics}

A total of 35 studies were selected from the initially retrieved 2,959 articles, which consisted of 39 retrospective cohorts (Figure 1). The sample size ranged from 30 to 285 , with an overall sample size of 3,980 cases. The chief source country of the included investigations is China $(n=13)$, followed by Japan $(n=4)$, South Korea $(n=4)$, and Spain $(n=4)$; Greece $(n=3)$; Germany $(n=2)$ and the United States $(n=2)$; and France $(n=1)$, Italy $(n=1)$, and the UK $(n=1)$. Breast cancer $(n=6)$, lung cancer $(n=5)$, renal cancer $(n=3)$, and pancreatic cancer $(n=3)$ were the most frequent cancer types in the included articles. A total of 30 studies reported overall survival, and 14 studies reported disease-free survival. Other detailed baseline features were summarized and recorded in Supplementary Table 1 [6-40].

\section{Methodological assessment}

Most of the included trials were graded as high quality in methodology, including 178 -score studies, 10 7-score studies, and 13 6-score studies. Only Florio et al. [9] were evaluated as a low-quality study, with a 4-score on the Newcastle-Ottawa Scale (Supplementary Table 1).

\section{Prognostic significance of p-4EBP1 in survival analysis}

Compared with patients who had high p-4EBP1 expression, patients with lower p-4EBP1 had a better outcome for 3 -year overall survival $(P<0.00001)$ 
(Figure 2). Our pooled analyses suggested that p-4EBP1 underexpression was a favorable indicator of 5-year overall survival among cancer patients, in contrast to those with redundant p-4EBP1 $(P<0.00001)$ (Figure 3$)$. Furthermore, there was a correlation between patients with higher p-4EBP1 positivity and limited 10-year overall survival $(P=0.03)$ (Figure 4).

Patients with underexpressed p-4EBP1 had a superior outcome of 3-year and 5-year disease- free survival compared with patients who had a higher expression $(P<0.0001$ and $P=0.0005)$. In contrast to patients with lower positivity, patients with p-4EBP1 overexpression obtained similar outcomes of 10-year disease-free survival $(P=0.64)$ (Supplementary Figure 1).

\section{Subgroup analyses}

\section{Tumor type}

Higher p-4EBP1 level indicated an unfavorable 3-year overall survival among patients with cholangiocarcinoma $(P=0.007)$, esophageal cancer $(P$ $=0.03)$, lung cancer $(P=0.03)$, nasopharyngeal cancer $(P=0.02)$, ovarian cancer $(P=0.0009)$, pancreatic cancer $(P=0.02)$, and other types $(P=0.02)$. However, patients with colorectal cancer $(P=0.84)$ displayed similar prognosis regardless of different p-4EBP1 expressions (Supplementary Figure 2). Similarly, p-4EBP1 underexpression was a beneficial indicator of 5 -year overall survival among patients with esophageal cancer $(P=0.0005)$, nasopharyngeal cancer $(P=0.0005)$, pancreatic cancer $(P=0.001)$, and other types $(P=0.01)$. Nevertheless, irrespective of colorectal cancer $(P=0.78)$ and lung cancer $(P=0.07)$, there was no significant difference in 5-year survival between the compared groups (Supplementary Figure 3).

Furthermore, p-4EBP1 isoform redundancy correlated to a worse 3-year disease-free survival in breast cancer $(P=0.003)$, renal cancer $(P=0.0004)$, and other types of cancer $(P=0.04)$. Additionally, underexpression of $\mathrm{p}$-4EBP1 predicted a longer 5-year disease-free survival in breast cancer $(P=0.008)$ and renal cancer $(P=0.004)$ but not in other types of cancer $(P=0.15)$ (Supplementary Figure 4).

\section{Source region}

All included studies were divided into Asian and non-Asian subgroups. Excessive p-4EBP1 expression was a negative indicator for 3 -year $(P=0.0001$ and $P=0.001)$ and 5-year $(P=0.0006$ and $P=0.01)$ overall survival

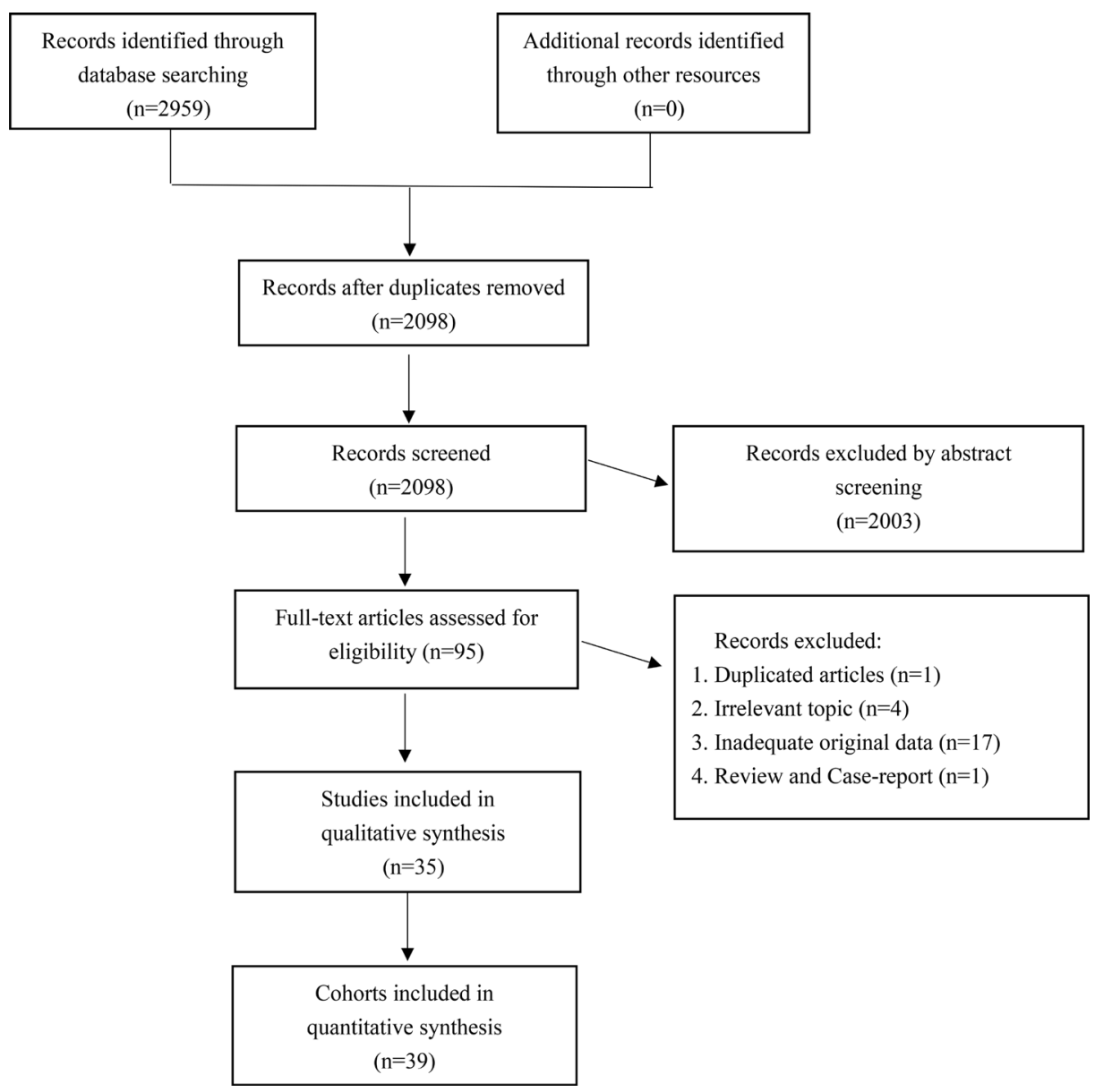

Figure 1: The selection flow chart of our meta-analysis. 
expectancy, both in Asian and in non-Asian subgroups (Supplementary Figures 5 and 6).

Sex

Overexpression of p-4EBP1 indicated a worse 3 -year and 5-year overall survival in the female-specific subgroup $(P<0.00001$ and $P=0.003)$. Similar results were found in the non-sex-specific subgroup $(P<0.0001$ and $P=0.0005)$ (Supplementary Figures 7 and 8 ).

\section{Mean age}

p-4EBP1 isoform redundancy was significantly linked to unfavorable prognosis among patients with mean age $<60$ years, irrespective of 3 -year $(P<0.0001)$ and 5-year $(P=0.0003)$ follow-up duration. A significant correlation exists between $\mathrm{p}-4 \mathrm{EBP} 1$ overexpression and 3 -year $(P=0.003)$ and 5-year $(P=0.02)$ overall survival among patients with mean age $>60$ years (Supplementary Figures 9 and 10).

\section{TNM stage}

Weaker p-4EBP1 positivity indicated longer 3-year and 5-year overall survival among patients with stage I-IV $(P=0.0005$ and $P=0.001)$, stage I-II $(P=0.0002$ and $P=0.002)$, stage II-IV $(P=0.01$ and $P=0.003)$, and stage II-IV $(P=0.0008$ and $P=0.003)$ cancers. However, patients with TNM stage IV had equivalent survival outcomes despite different p-4EBP1 expression $(P=0.71$ and $P=0.48$ ) (Supplementary Figures 11 and 12).

\section{Phosphorylation site}

Underexpression of p-4EBP1 in phosphorylation sites threonine $37 / 46(\mathrm{Thr} 37 / 46)(P=0.0008)$ threonine 70 (Thr70) $(P=0.02)$ and serine $65($ Ser65) $(P=0.02)$ indicated prolonged 3-year overall survival among patients with malignancies. However, although high p-4EBP1 expression in phosphorylation sites Thr37/46 $(P=0.0006)$ and Ser65 $(P=0.0004)$ had a favorable outcome for 5-year overall survival, Thr70 had equivalent 5-year overall survival despite a different p-4EBP1 expression $(P=0.14)$ (Supplementary Figures 13 and 14).

\section{Subcellular localization}

In subgroups characterized by different subcellular localization, our quantitative results suggested that trials based on cytoplasmic staining of p-4EBP1 correlated its over-reactivity with a worse 3 -year $(P=0.03)$ overall survival but not with a worse 5-year overall survival $(P=0.15)$. There was no significant correlation between underexpression of nuclear p-4EBP1 and long-term prognosis among cancer patients (3-year overall survival: $P=0.27$; 5-year overall survival: $P=0.25$ ), although a significant correlation exists between overexpression of total p-4EBP1 and 3-year $(P<0.00001)$ and 5-year

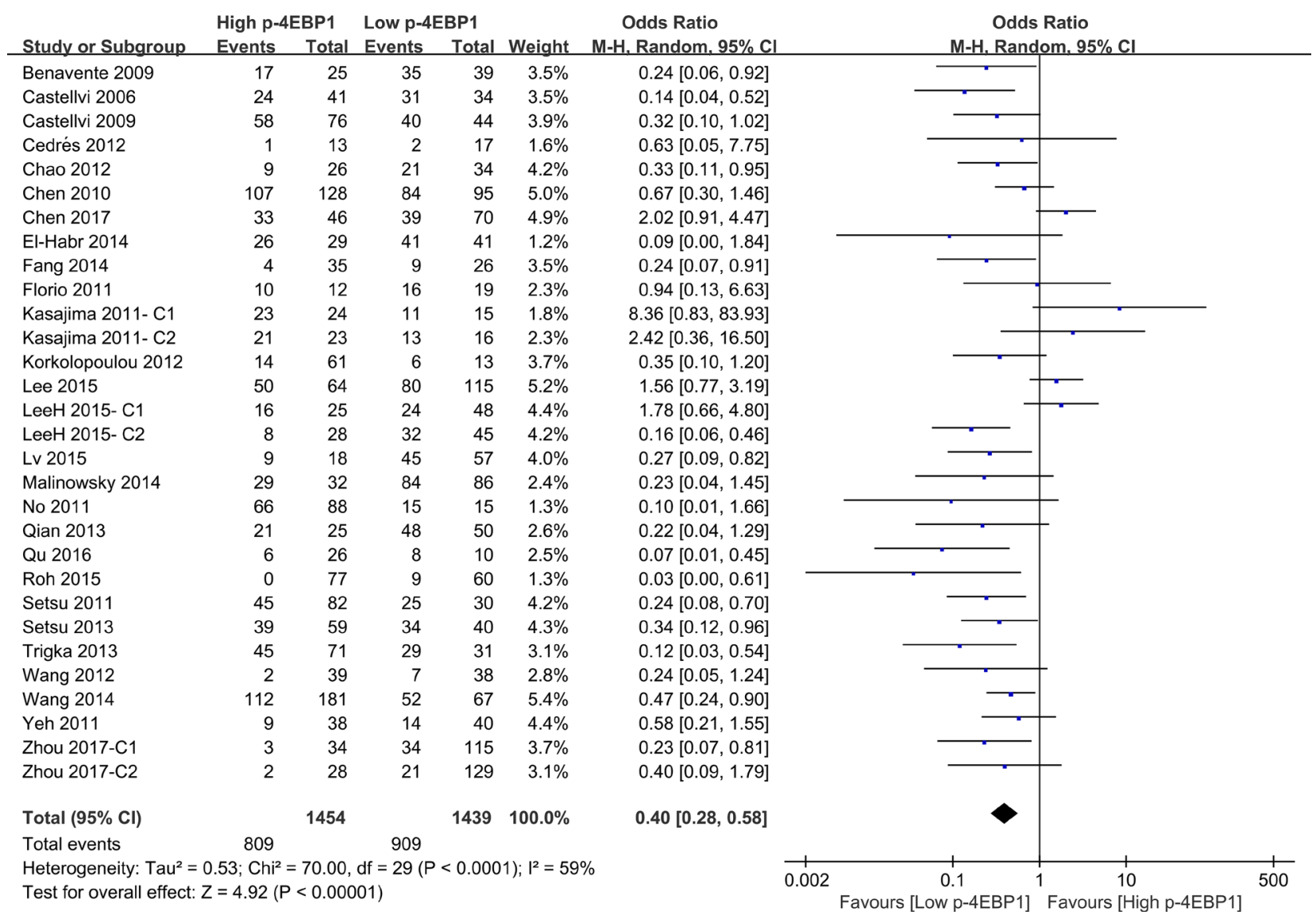

Figure 2: The correlation between p-4EBP1 expression and 3-year overall survival in malignancies. 
$(P<0.0001)$ overall survival (Supplementary Figures 15 and 16).

\section{Sensitivity analysis}

First, by excluding low-quality trials of Di Florio et al.[9], outcomes of 3-year $(P<0.00001)$, 5-year $(P<0.0001)$, and 10-year $(P=0.05)$ overall survival and 3-year $(P<0.0001)$, 5-year $(P=0.0005)$, and 10-year $(P=0.64)$ disease-free survival remained stable.

Second, by interchanging statistical modes between a fixed-effects model and a random-effects model, outcomes of 3-year, 5-year, and 10-year overall survival and 3-year, 5-year, and 10-year disease-free survival remained unchanged.
Third, by randomly eliminating included trials on the STATA 14.0 platform, the outcome stability of 3-year and 5-year overall survival and 3-year, 5-year, and 10-year disease-free survival was graphically confirmed. However, the result of 10-year overall survival seems unstable (Supplementary Figure 17).

\section{Publication bias}

The funnel plots of 3-year overall survival and disease-free survival were graphically symmetric. Additionally, the Harbord test $(P=0.139$ and $P=0.192)$ and the Peters test $(P=0.554$ and $P=0.357)$ confirmed that no publication bias existed among the included studies (Supplementary Figure 18).

\begin{tabular}{|c|c|c|c|c|c|}
\hline \multirow[b]{2}{*}{ Study or Subgroup } & \multicolumn{2}{|c|}{ High p-4EBP1 } & \multicolumn{2}{|c|}{ Low p-4EBP1 } & \multirow[b]{2}{*}{ Weight } \\
\hline & Events & Total & Events & Total & \\
\hline Benavente 2009 & 12 & 25 & 29 & 39 & $4.5 \%$ \\
\hline Castellvi 2006 & 6 & 41 & 31 & 34 & $3.8 \%$ \\
\hline Castellvi 2009 & 57 & 76 & 40 & 44 & $4.4 \%$ \\
\hline Chao 2012 & 4 & 26 & 20 & 34 & $4.2 \%$ \\
\hline Chen 2010 & 77 & 128 & 72 & 95 & $5.3 \%$ \\
\hline Chen 2017 & 25 & 46 & 32 & 70 & $5.1 \%$ \\
\hline El-Habr 2014 & 26 & 29 & 41 & 41 & $1.9 \%$ \\
\hline Fang 2014 & 0 & 35 & 4 & 26 & $1.9 \%$ \\
\hline Florio 2011 & 7 & 12 & 16 & 19 & $3.5 \%$ \\
\hline Kasajima 2011- C1 & 21 & 24 & 9 & 15 & $3.6 \%$ \\
\hline Kasajima 2011- C2 & 21 & 23 & 10 & 16 & $3.3 \%$ \\
\hline Korkolopoulou 2012 & 0 & 61 & 6 & 13 & $1.9 \%$ \\
\hline Lee 2015 & 50 & 64 & 67 & 115 & $5.1 \%$ \\
\hline LeeH 2015- C1 & 12 & 25 & 17 & 48 & $4.7 \%$ \\
\hline LeeH 2015- C2 & 6 & 28 & 24 & 45 & $4.5 \%$ \\
\hline Lv 2015 & 3 & 18 & 40 & 57 & $4.0 \%$ \\
\hline Malinowsky 2014 & 27 & 32 & 80 & 86 & $4.2 \%$ \\
\hline Qian 2013 & 18 & 25 & 44 & 50 & $4.3 \%$ \\
\hline Qu 2016 & 0 & 26 & 8 & 10 & $1.8 \%$ \\
\hline Setsu 2011 & 39 & 82 & 20 & 30 & $4.9 \%$ \\
\hline Setsu 2013 & 30 & 59 & 31 & 40 & $4.8 \%$ \\
\hline Trigka 2013 & 45 & 71 & 29 & 31 & $3.8 \%$ \\
\hline Wang 2014 & 98 & 181 & 48 & 67 & $5.3 \%$ \\
\hline Yeh 2011 & 5 & 38 & 13 & 40 & $4.4 \%$ \\
\hline Zhou 2017-C1 & 1 & 34 & 34 & 115 & $3.0 \%$ \\
\hline Zhou 2017-C2 & 0 & 28 & 18 & 129 & $2.0 \%$ \\
\hline Total $(95 \% \mathrm{Cl})$ & & 1237 & & 1309 & $100.0 \%$ \\
\hline Total events & 590 & & 783 & & \\
\hline
\end{tabular}

Odds Ratio

. Random, $95 \% \mathrm{Cl}$ $0.32[0.11,0.92]$ $0.02[0.00,0.07]$ $0.30[0.09,0.95]$ $0.13[0.04,0.45]$ $0.48[0.27,0.87]$ $1.41[0.67,2.98]$ $0.09[0.00,1.84]$ $0.07[0.00,1.37]$ $0.26[0.05,1.41]$ $4.67[0.95,22.90]$ $6.30[1.07,36.94]$ $0.01[0.00,0.18]$ $2.56[1.27,5.15]$ $1.68[0.63,4.50]$ $0.24[0.08,0.70]$ $0.09[0.02,0.33]$ $0.41[0.11,1.43]$ $0.35[0.10,1.19]$ $0.01[0.00,0.13]$ $0.45[0.19,1.09]$ $0.30[0.12,0.74]$ $0.12[0.03,0.54]$ $0.47[0.25,0.86]$ $0.31[0.10,0.99]$ $0.07[0.01,0.55]$ $0.11[0.01,1.81]$

$0.32[0.19,0.53]$

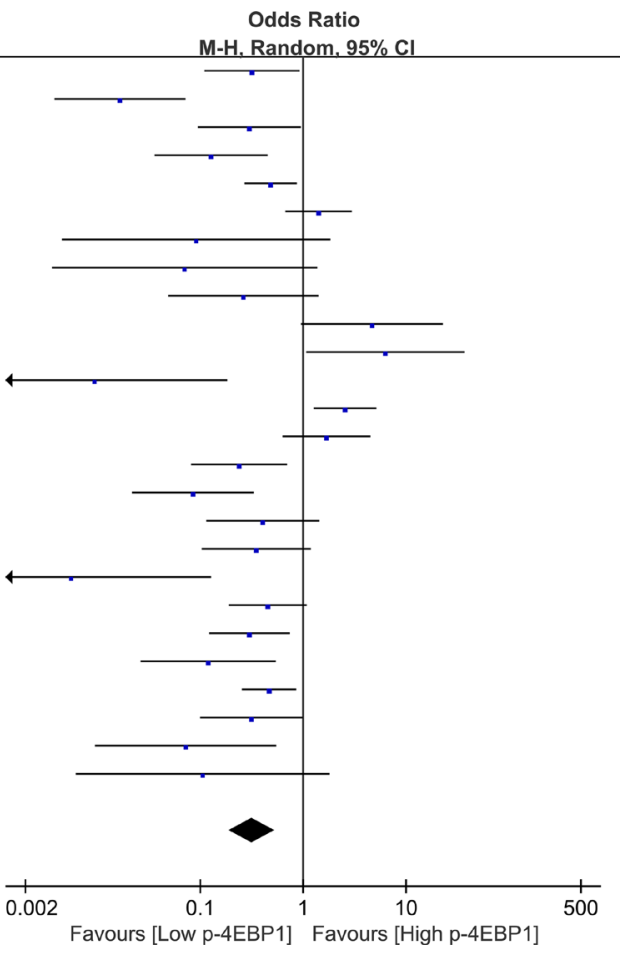

Figure 3: The correlation between p-4EBP1 expression and 5-year overall survival in malignancies.

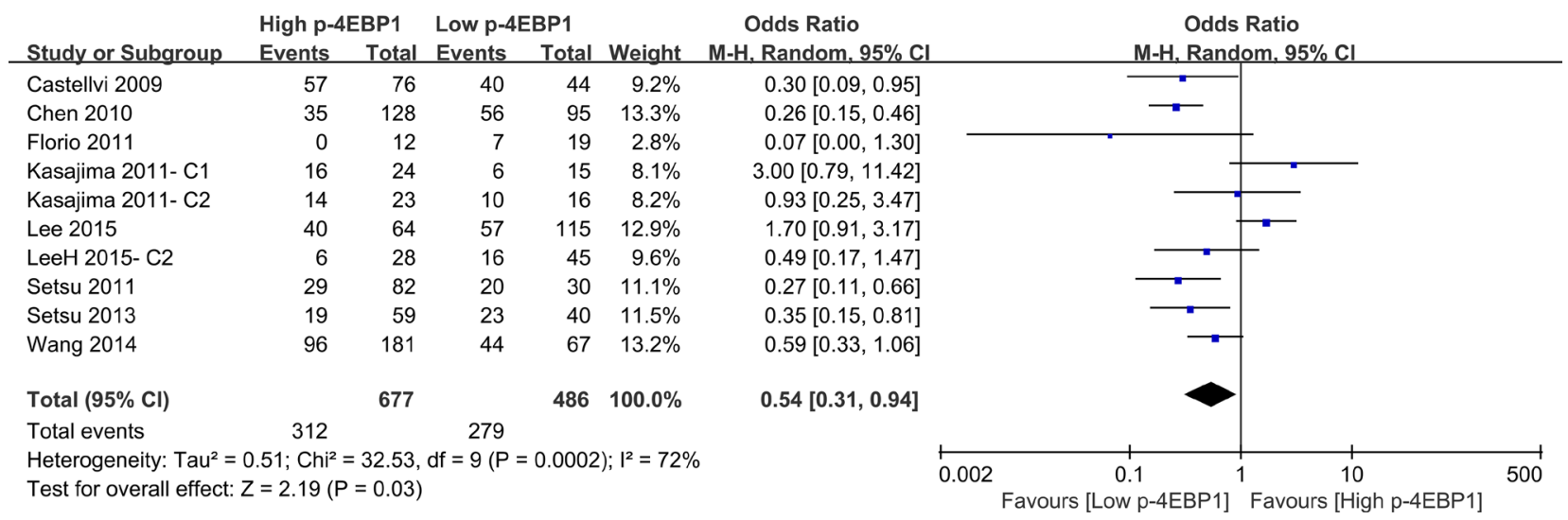

Figure 4: The correlation between p-4EBP1 expression and 10-year overall survival in malignancies. 


\section{DISCUSSION}

mTOR, a highly conserved serine/threonine protein kinase, promotes cell growth and proliferation through phosphorylation of 4EBP1 and S6k, thus controlling protein translation [41]. Evidence indicates that the disruption of protein synthesis at the level of 4EBP1/eIF4E downstream of mTORC1 stimulates tumor formation. Suppression of 4EBP1 activity and the accompanying activation of capdependent translation promotes cell proliferation and correlates with cancer development [42]. p-4EBP1, the inactivated form of 4EBP1, might act as a tumor promoter by converging upstream oncogenic signals and driving the proliferative signal downstream, initiating the synthesis of oncogenic proteins such as cyclin D1, c-myc, or vascular endothelial growth factor (VEGF) [43, 44].

Our quantitative results show that higher expression of p-4EBP1 indicates unfavorable prognosis among cancer patients, irrespective of 3-year, 5-year, and 10-year overall survival and 3-year and 5-year disease-free survival. The present study is the first meta-analysis to systemically study the possible prognostic value of $\mathrm{p}-4 \mathrm{EBP} 1$ overexpression in human malignancies. However, our results showing no obvious difference between 10-year disease-free survival, which is the limit of follow-up duration of many studies, and leading to the insufficient number of cohorts $(n=$ 4) and participants $(n=621)$ might be the reasonable interpretation of this puzzling consequence. Furthermore, in a departure from the primary included cohorts, Azim et al. [8], Chen et al. [16], Kasajima et al. [19], Lee et al. [7], and Lee et al. [21] suggested that the prognostic value of p-4EBP1 level among cancer patients remains blurred. A significant difference of TNM stage existed between the two groups in Chen et al. [16] $(P=0.001)$ and Lee et al [7] $(P<0.001)$, and the underexpression group had more advanced TNM stages. Whether these outcomes are simply exceptions or were distorted by the confounding element of different TNM stages awaits further clarification. Moreover, according to Azim et al. [8], the adjuvant treatment received and small number of cases might be reasonable explanations for the different results. Lee et al [21] and Kasajima et al. [19] suggest that all outcomes might result from the small number of participants included in these studies, so the results might be confirmed in largescale prospective studies.

In-depth perspectives of the negative prognostic significance of $\mathrm{p}$-4EBP1 were supplied by subgroup analyses. In different tumor types, underexpression of p-4EBP1 consistently acted as an indicator of better prognosis among patients with breast cancer, cholangiocarcinoma, esophageal cancer, nasopharyngeal cancer, ovarian cancer, pancreatic cancer, and renal cancer. The prognostic impact of p-4EBP1 on colorectal cancer remains ambiguous. Our pooled results confirmed that lower p-4EBP1 level indicates a better survival prognosis regardless of source region, sex, or mean age. Our outcome shows that the prognostic value of p-4EBP1 overexpression was not distorted despite the variety of TNM stages, except for TNM IV, which means that p-4EBP1 might participate in early phases of tumorigenesis and cancer progression. Total and cytoplasmic p-4EBP1 was verified to correlate with a worse prognosis, whereas nuclear expression was not correlated with an unfavorable outcome prediction. This disparity is probably attributable to the fact that mTOR is predominantly cytoplasmic and phosphorylates 4EBP1 in cytoplasm [45]. When 4EBP1 is phosphorylated, the binding affinity for eIF4E is reduced, which promotes the dissociation of eIF4E from p-4EBP1, attenuating the inhibitory effect on eIF4E-dependent translation initiation, including some oncogenes [46].

Although our meta-analysis was designed and performed rigorously, it has limitations. First, all included cohorts were in retrospective studies, which might cause higher internal heterogeneity. The internal heterogeneity might be explained by the different patient characteristics and the different tumor types or TNM stages, such as in Chen et al. [16] and Lee et al. [7]. Prospective and randomized controlled trials are thus needed for a more persuasive conclusion in future studies. Second, although the total sample size reached 3,980 , the included cases were still insufficient to draw a consolidated result, especially for the subgroup analyses and 10-year overall survival, which seems unstable according to sensitivity analysis. In addition, as shown in Figure 1, 17 studies related to p-4EBP1 expression and malignancy prognosis were excluded by our meta-analysis because of the lack of original data. Five of the studies indicated that the p-4EBP1 level had a significant impact on cancer prognosis [47-51], but four studies indicated no impact [52-55]. Another limitation is that bias might be introduced because the survival data were mainly extracted from Kaplan-Meier curves and digitized by GetData Graph Digitizer 2.25 software.

This meta-analysis is the first that confirms the unfavorable influence of p-4EBP1 overexpression in survival expectancy. It correlates with worse prognosis for 3-year and 5-year overall and disease-free survival among cancer patients. Additionally, the prognostic significance is independent of sex, mean age, and resource region. Also, p-4EBP1 Thr37/46 instead of p-4EBP1 Thr70, and total p-4EBP1 but not cytoplasmic or nuclear-positive p-4EBP1, act as prognostic predictors in most cancers, which provide more precise data to guide the clinical evaluations for diagnosis and prognosis. Therefore, we believe that p-4EBP1-directed therapy is a hopeful strategy for cancer patients.

\section{ACKNOWLEDGMENTS}

We sincerely thank all staff in our department for offering methodological assistance. 


\section{CONFLICTS OF INTEREST}

The authors declare no conflicts of interest.

\section{FUNDING}

This meta-analysis was financially supported by the Natural Science Foundation of China (NO. 81570568).

\section{REFERENCES}

1. $\mathrm{Xu} \mathrm{K}$, Liu P, Wei W. mTOR signaling in tumorigenesis. Biochim Biophys Acta. 2014; 2:638-54.

2. She QB. 4E-BP1 as an oncotarget. Aging (Albany NY). 2015; 7:517-18. https://doi.org/10.18632/aging.100794.

3. Huang K, Fingar DC. Growing knowledge of the mTOR signaling network. Semin Cell Dev Biol. 2014; 79-90.

4. Zoncu R, Efeyan A, Sabatini DM. mTOR: from growth signal integration to cancer, diabetes and ageing. Nat Rev Mol Cell Biol. 2011; 1:21-35.

5. Cai W, Ye Q, She QB. Loss of 4E-BP1 function induces EMT and promotes cancer cell migration and invasion via cap-dependent translational activation of snail. Oncotarget. 2014; 15:6015-27. https://doi.org/10.18632/ oncotarget.2109.

6. Benavente S, Vergés R, Hermosilla E, Fumanal V, Casanova N, García A, Ramón Y Cajal S, Giralt J. Overexpression of phosphorylated 4E-BP1 predicts for tumor recurrence and reduced survival in cervical carcinoma treated with postoperative radiotherapy. Int J Radiat Oncol Biol Phys. 2009; 5:1316-22.

7. Lee HW, Park MI, Kim MS, Kim SH, Roh MS, Kim K, Jung SB, Lee EH. Overexpression of phosphorylated 4E-binding protein 1 and its clinicopathological significances in gastric cancer. Pathol Res Pract. 2015; 4:298-302.

8. Azim HA, Kassem L, Treilleux I, Wang Q, El Enein MA, Anis SE, Bachelot T. Analysis of PI3K/mTOR Pathway Biomarkers and Their Prognostic Value in Women with Hormone Receptor-Positive, HER2-Negative Early Breast Cancer. Transl Oncol. 2016; 2:114-23.

9. Di Florio A, Adesso L, Pedrotti S, Capurso G, Pilozzi E, Corbo V, Scarpa A, Geremia R, Delle Fave G, Sette C. Src kinase activity coordinates cell adhesion and spreading with activation of mammalian target of rapamycin in pancreatic endocrine tumour cells. Endocr Relat Cancer. 2011; 5:541-54.

10. Campbell L, Jasani B, Griffiths DF, Gumbleton M. Phospho-4e-BP1 and eIF4E overexpression synergistically drives disease progression in clinically confined clear cell renal cell carcinoma. Am J Cancer Res. 2015; 9:2838-2848.

11. Castellvi J, Garcia A, Rojo F, Ruiz-Marcellan C, Gil A, Baselga J, Ramon y Cajal S. Phosphorylated 4E binding protein 1: a hallmark of cell signaling that correlates with survival in ovarian cancer. Cancer. 2006; 8:1801-11.

12. Castellvi J, Garcia A, Ruiz-Marcellan C, Hernandez-Losa J,
Peg V, Salcedo M, Gil-Moreno A, Ramon y Cajal S. Cell signaling in endometrial carcinoma: phosphorylated 4E-binding protein-1 expression in endometrial cancer correlates with aggressive tumors and prognosis. Hum Pathol. 2009; 10:1418-26.

13. Cedres S, Montero MA, Martinez P, Martinez A, Rodriguez-Freixinos V, Torrejon D, Gabaldon A, Salcedo M, Ramon Y, Cajal S, Felip E. Exploratory analysis of activation of PTEN-PI3K pathway and downstream proteins in malignant pleural mesothelioma (MPM). Lung Cancer. 2012; 1:192-8.

14. Chao YK, Chuang WY, Yeh CJ, Chang YS, Wu YC, Kuo SY, Hsieh MJ, Hsueh C. High phosphorylated 4E-binding protein 1 expression after chemoradiotherapy is a predictor for locoregional recurrence and worse survival in esophageal squamous cell carcinoma patients. J Surg Oncol. 2012; 3:288-92.

15. Chen J, Hu CF, Hou JH, Shao Q, Yan LX, Zhu XF, Zeng YX, Shao JY. Epstein-Barr virus encoded latent membrane protein 1 regulates mTOR signaling pathway genes which predict poor prognosis of nasopharyngeal carcinoma. J Transl Med. 2010; 30.

16. Chen YT, Tsai HP, Wu CC, Wang JY, Chai CY. Eukaryotic translation initiation factor 4E (eIF-4E) expressions are associated with poor prognosis in colorectal adenocarcinoma. Pathol Res Pract. 2017; 5:490-5.

17. El-Habr EA, Levidou G, Trigka EA, Sakalidou J, Piperi C, Chatziandreou I, Spyropoulou A, Soldatos R, Tomara G, Petraki K, Samaras V, Zisakis A, Varsos V, et al. Complex interactions between the components of the PI3K/AKT/ mTOR pathway, and with components of MAPK, JAK/ STAT and Notch-1 pathways, indicate their involvement in meningioma development. Virchows Arch. 2014; $4: 473-85$.

18. Fang Z, Lu L, Tian Z, Luo K. Overexpression of phosphorylated 4E-binding protein 1 predicts lymph node metastasis and poor prognosis of Chinese patients with hilar cholangiocarcinoma. Med Oncol. 2014; 31:940.

19. Kasajima A, Pavel M, Darb-Esfahani S, Noske A, Stenzinger A, Sasano H, Dietel M, Denkert C, Rocken C, Wiedenmann B, Weichert W. mTOR expression and activity patterns in gastroenteropancreatic neuroendocrine tumours. Endocr Relat Cancer. 2011; 1:181-92.

20. Korkolopoulou P, Levidou G, El-Habr EA, Piperi C, Adamopoulos C, Samaras V, Boviatsis E, Thymara I, Trigka EA, Sakellariou S, Kavantzas N, Patsouris E, Saetta AA. Phosphorylated 4E-binding protein 1 (p-4E-BP1): a novel prognostic marker in human astrocytomas. Histopathology. $2012 ; 2: 293-305$.

21. Lee HW, Lee EH, Lee JH, Kim JE, Kim SH, Kim TG, Hwang SW, Kang KW. Prognostic significance of phosphorylated 4E-binding protein 1 in non-small cell lung cancer. Int J Clin Exp Pathol. 2015; 4:3955-62.

22. Lv T, Wang Q, Cromie M, Liu H, Tang S, Song Y, Gao W. Twist1-mediated 4E-BP1 regulation through $\mathrm{mTOR}$ in 
non-small cell lung cancer. Oncotarget. 2015; 32:33006-18. https://doi.org/10.18632/oncotarget.5026.

23. Ma BL, Shan MH, Sun G, Ren GH, Dong C, Yao X, Zhou M. Immunohistochemical analysis of phosphorylated mammalian target of rapamycin and its downstream signaling components in invasive breast cancer. Mol Med Rep. 2015; 4:5246-54.

24. Malinowsky K, Nitsche U, Janssen KP, Bader FG, Spath C, Drecoll E, Keller G, Hofler H, Slotta-Huspenina J, Becker KF. Activation of the PI3K/AKT pathway correlates with prognosis in stage II colon cancer. Br J Cancer. 2014; 110:2081-9.

25. Meric-Bernstam F, Chen H, Akcakanat A, Do KA, Lluch A, Hennessy BT, Hortobagyi GN, Mills GB, Gonzalez-Angulo A. Aberrations in translational regulation are associated with poor prognosis in hormone receptor-positive breast cancer. Breast Cancer Res. 2012; 14:R138.

26. Nishikawa $M$, Miyake $H$, Harada $K$, Fujisawa $M$. Expression of molecular markers associated with the mammalian target of rapamycin pathway in nonmetastatic renal cell carcinoma: Effect on prognostic outcomes following radical nephrectomy. Urol Oncol. 2014; 1:49. e15-21.

27. Nishikawa M, Miyake H, Behnsawy HM, Fujisawa M. Significance of 4E-binding protein 1 as a therapeutic target for invasive urothelial carcinoma of the bladder. Urol Oncol. 2015; 4:166.e9-15.

28. No JH, Jeon YT, Park IA, Kim YB, Kim JW, Park NH, Kang SB, Han JY, Lim JM, Song YS. Activation of mTOR signaling pathway associated with adverse prognostic factors of epithelial ovarian cancer. Gynecol Oncol. 2011; 1:8-12.

29. Qian ZR, Ter-Minassian M, Chan JA, Imamura Y, Hooshmand SM, Kuchiba A, Morikawa T, Brais LK, Daskalova A, Heafield R, Lin X, Christiani DC, Fuchs CS, et al. Prognostic significance of MTOR pathway component expression in neuroendocrine tumors. J Clin Oncol. 2013; 27:3418-25.

30. Qu Y, Ye D, Zhang H, Shi G, Zhu Y, Dai B, Wang H. Phosphorylated 4EBP1 is associated with tumor progression and poor prognosis in Xp11.2 translocations renal cell carcinoma. Sci Rep. 2016; 6:23594.

31. Roh MS, Lee JH, Kang KW, Nam HY, Jung SB, Kim K, Lee EH, Park MI, Kim MS, Lee HW. Phosphorylated 4E-binding protein 1 expression is associated with poor prognosis in small-cell lung cancer. Virchows Arch. 2015; 6:667-73.

32. Setsu N, Yamamoto H, Kohashi K, Endo M, Matsuda S, Yokoyama R, Nishiyama K, Iwamoto Y, Dobashi Y, Oda Y. The Akt/mammalian target of rapamycin pathway is activated and associated with adverse prognosis in soft tissue leiomyosarcomas. Cancer. 2012; 6:1637-48.

33. Setsu N, Kohashi K, Fushimi F, Endo M, Yamamoto H, Takahashi Y, Yamada Y, Ishii T, Yokoyama K, Iwamoto Y, Oda Y. Prognostic impact of the activation status of the
Akt/mTOR pathway in synovial sarcoma. Cancer. 2013; 19:3504-13.

34. Trigka EA, Levidou G, Saetta AA, Chatziandreou I, Tomos P, Thalassinos N, Anastasiou N, Spartalis E, Kavantzas N, Patsouris E, Korkolopoulou P. A detailed immunohistochemical analysis of the PI3K/AKT/mTOR pathway in lung cancer: correlation with PIK3CA, AKT1, K-RAS or PTEN mutational status and clinicopathological features. Oncol Rep. 2013; 2:623-36.

35. Wang $\mathrm{Z}$, Zheng $\mathrm{T}$, Wu Q, Wang $\mathrm{J}$, Wu $\mathrm{C}$, Wang $\mathrm{J}$. Immunohistochemical analysis of the mTOR pathway in intrahepatic cholangiocarcinoma. Neoplasma. 2012; 2:137-41.

36. Wang W, Wen Q, Xu L, Xie G, Li J, Luo J, Chu S, Shi L, Huang D, Li J, Fan S. Activation of Akt/mTOR pathway is associated with poor prognosis of nasopharyngeal carcinoma. PLoS One. 2014; 9:e106098.

37. Wang S, Sun Y, He A, Zheng C, Zheng X. Predictive value of phosphorylated mammalian target of rapamycin for disease-free survival in breast cancer patients receiving neoadjuvant chemotherapy. Oncol Lett. 2014; 6:2642-8.

38. Yeh CJ, Chuang WY, Chao YK, Liu YH, Chang YS, Kuo SY, Tseng CK, Chang HK, Hsueh C. High expression of phosphorylated 4E-binding protein 1 is an adverse prognostic factor in esophageal squamous cell carcinoma. Virchows Arch. 2011; 2:171-8.

39. Zhou X, Tan M, Stone Hawthorne V, Klos KS, Lan KH, Yang Y, Yang W, Smith TL, Shi D, Yu D. Activation of the Akt/mammalian target of rapamycin/4E-BP1 pathway by ErbB2 overexpression predicts tumor progression in breast cancers. Clin Cancer Res. 2004; 20:6779-88.

40. Zhou L, Yuan D, Zhang ZG, Liang ZY, Zhou WX, Yang JY, Jiang SH, Lu J, Zhang TP, You L, Guo JC, Zhao YP. Expression of key mTOR pathway components in pancreatic ductal adenocarcinoma: A multicenter study for clinicopathologic and prognostic significance. Cancer Lett. 2017; 395:45-52.

41. Cargnello M, Tcherkezian J, Roux PP. The expanding role of mTOR in cancer cell growth and proliferation. Mutagenesis. 2015; 2:169-76.

42. Laplante M, Sabatini DM. mTOR signaling in growth control and disease. Cell. 2012; 2:274-93.

43. Armengol G, Rojo F, Castellví J, Iglesias C, Cuatrecasas M, Pons B, Baselga J, Ramón y Cajal S. 4E-Binding Protein 1: A Key Molecular "Funnel Factor" in Human Cancer with Clinical Implications. Cancer Res. 2007; 16:7551-7555.

44. Averous J, Proud CG. When translation meets transformation: The mTOR story. Oncogene. 2006; 48:6423-35.

45. Guertin DA, Sabatini DM. Defining the role of mTOR in cancer. Cancer Cell. 2007; 1:9-22.

46. Armengol G, Rojo F, Castellvi J, Iglesias C, Cuatrecasas M, Pons B, Baselga J, Ramon y Cajal S. 4E-binding protein 1: a key molecular "funnel factor" in human cancer with clinical implications. Cancer Res. 2007; 16:7551-5. 
47. Graff JR, Konicek BW, Lynch RL, Dumstorf CA, Dowless MS, McNulty AM, Parsons SH, Brail LH, Colligan BM, Koop JW, Hurst BM, Deddens JA, Neubauer BL, et al. eIF4E activation is commonly Elevated in advanced human prostate cancers and significantly related to reduced patient survival. Cancer Res. 2009; 9:3866-73.

48. Lakiotaki E, Levidou G, Angelopoulou MK, Adamopoulos C, Pangalis G, Rassidakis G, Vassilakopoulos T, Gainaru G, Flevari P, Sachanas S, Saetta AA, Sepsa A, Moschogiannis $\mathrm{M}$, et al. Potential role of AKT/mTOR signalling proteins in hairy cell leukaemia: association with BRAF/ERK activation and clinical outcome. Sci Rep. 2016; 21252.

49. Millican-Slater RA, Sayers CD, Hanby AM, Hughes TA. Expression of phosphorylated eIF4E-binding protein 1, but not of eIF4E itself, predicts survival in male breast cancer. Br J Cancer. 2016; 3:339-45.

50. Mueller S, Phillips J, Onar-Thomas A, Romero E, Zheng S, Wiencke JK, McBride SM, Cowdrey C, Prados MD, Weiss WA, Berger MS, Gupta N, Haas-Kogan DA. PTEN promoter methylation and activation of the PI3K/Akt/ mTOR pathway in pediatric gliomas and influence on clinical outcome. Neuro Oncol. 2012; 9:1146-52.
51. Miao Y, Chen L, Shi C, Fan R, Chen P, Liu H, Xia A, Qian H. Increased phosphorylation of 4Ebinding protein 1 predicts poor prognosis for patients with colorectal cancer. Mol Med Rep. 2017; 5:3099-104.

52. Kim SJ, Kim JH, Jung HS, Lee TJ, Lee KM, Chang IH. Phosphorylated p70S6K in noninvasive low-grade urothelial carcinoma of the bladder: correlation with tumor recurrence. Asian J Androl. 2014; 4:611-7.

53. Nakai Y, Miyake M, Morizawa Y, Hori S, Tatsumi Y, Anai S, Onishi S, Tanaka N, Fujimoto K. Potential biomarkers for the therapeutic efficacy of sorafenib, sunitinib and everolimus. Oncol Rep. 2017; 1:227-34.

54. Park SJ, Lee TJ, Chang IH. Role of the mtor pathway in the progression and recurrence of bladder cancer: An immunohistochemical tissue microarray study. Korean $\mathrm{J}$ Urol. 2011; 7:466-73.

55. Munari E, Fujita K, Faraj S, Chaux A, Gonzalez-Roibon N, Hicks J, Meeker A, Nonomura N, Netto GJ. Dysregulation of mammalian target of rapamycin pathway in upper tract urothelial carcinoma. Hum Pathol. 2013; 12:2668-76. 